\title{
X-Ray Tomography for Structural Analysis of Microstructured Optical Fibres and Preforms
}

\author{
S. R. Sandoghchi ${ }^{(1)}$, G. T. Jasion ${ }^{(1)}$, N. V. Wheeler ${ }^{(1)}$, J. P. Wooler $^{(1)}$, R. P. Boardman ${ }^{(2)}$, N. Baddela(1) \\ Y. Chen ${ }^{(1)}$, J. Hayes ${ }^{(1)}$, E. Numkam Fokoua ${ }^{(1)}$, T. Bradley $^{(1)}$, D. R. Gray $y^{(1)}$, S. M. Mousavi ${ }^{(1)}$, M. \\ Petrovich $^{(1)}$, F. Poletti $^{(1)}$, and D. J. Richardson ${ }^{(1)}$ \\ (1) Optoelectronics Research Centre, University of Southampton, Southampton SO17 1BJ, UK, \\ srs1g12@soton.ac.uk \\ ${ }^{(2)} \mu$-VIS Centre for Computer Tomography, University of Southampton, Southampton SO17 1BJ, UK.
}

\begin{abstract}
X-ray computational tomography is demonstrated as a powerful non-destructive tool to image the internal structure of a hollow core photonic band-gap fibre and its preforms. The technique is applied to measure the deformation within a splice with unprecedented detail.
\end{abstract}

\section{Introduction}

The consistency of the longitudinal and crosssectional structure of microstructured optical fibre's (MOFs) preforms determines the stability of the fabrication process and the quality of the fabricated fibres. Structural analysis of a fibre along its length, on the other hand, provides information about the fabrication process and its preform. However, determining the origin of inconsistencies found in fibres and associating them with problems in the preform or in the fabrication process is extremely difficult, if not impossible, without a detailed knowledge of the quality of the preform. Preform analysis should ideally be non-destructive to reduce cost and, more importantly, to enable fibre fabrication to be performed from the same preform, thus improving the ability to identify correlations between anomalies in the preform and in the fibre. In many cases, such correlation analysis presents very substantial challenges.

In this work we present the first use of X-ray computational tomography (CT) to image the internal structure of hollow-core photonic bandgap fibres (HC-PBGFs) and their preforms in a non-destructive fashion. In addition, we demonstrate application of the technique to understand the internal deformations caused during the splice process of HC-PBGFs.

\section{Structural non-uniformities}

Optical and electron microscopy are the most common techniques of structural inspection of fibre optics. These techniques require clear line of sight to the target under inspection. Therefore, to image the internal structure of the fibre or preform, one should create a flat cross section (e.g. by cleaving). Cutting the preform is clearly undesirable, as the preform could no longer be used. Additionally, for some fibre types achieving a clean cleave is difficult, as is the case with multi-element fibre ${ }^{1}$ and metal-rod incorporated fibres ${ }^{2}$. Furthermore, in the case of a rapidly changing structure, such as a longitudinal taper, splice point, or defective point along the fibre, the cleaving approach ultimately limits the spatial resolution at which the structural change can be analysed ${ }^{3}$ as the number of sequential cuts per unit length is limited in practice to $\sim 0.4 / \mathrm{mm}$. Moreover, the presence of orientation dependent features, such as twist, are difficult to capture using sequential cleaves.

X-ray CT is a method to obtain three dimensional density data of a sample. Conventional $2 \mathrm{D}$ radiographs capture the $\mathrm{X}$-ray density of the sample from hundreds of different angles. The data is then reconstructed into a 3D data set with each voxel (pixel in 3D space) containing a value of the X-ray density of that location in space. This method can capture complex internal geometries where there is sufficient contrast between material densities ${ }^{4}$.

$X$-ray CT is used prominently in medical applications but is finding increasing scientific applications in the fields of material science, archaeology and marine biology. Recent advances in this field ${ }^{5}$ allow voxel sizes smaller than $50 \mathrm{~nm}$.

In this work the X-ray CT scans were performed at the $\mu$-VIS centre at the University of Southampton using 225kV Nikon HMX ST and Zeiss Xradia Versa 510 machines. In this paper, we provide the first demonstration of the potential of X-ray CT for both preform and fibre analysis, showing that it enables non-destructive measurements that simply cannot be made by other means.

\section{Hollow Core Photonic Band-Gap Fibres}

HC-PBGFs are a bold alternative to conventional optical fibre for the next generation of optical networks, offering ultra-low nonlinearity, ultimate latency and the prospect of ultra-low loss ${ }^{6}$. They consist of a microstructured cladding with sub$\mu \mathrm{m}$ nodes connected by very thin glass membranes extended along the length of fibre.

HC-PBGFs are fabricated through a complex 
a)

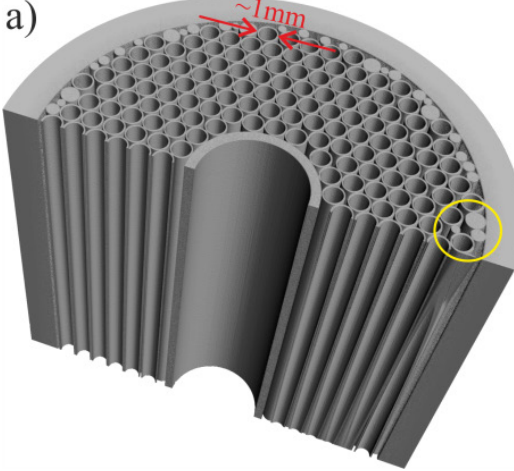

b)

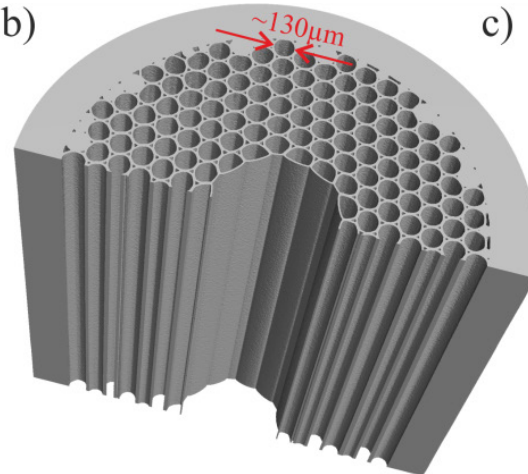

c)

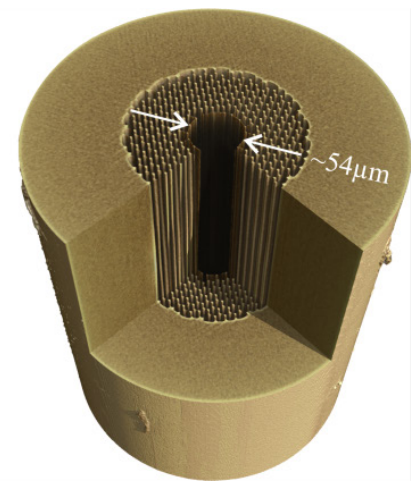

Fig. 1. Images from X-ray CT scans, left to right: a) $1^{\text {st }}$ stage preform of a 37-cell hollow core incorporating a core tube with capillary arrangement drift highlighted, b) cane of a 19-cell hollow core and c) a 19-cell hollow core photonic band gap fibre. manual fabrication process in which, a plurality of $\mathrm{mm}$ size high purity glass capillaries are stacked in a hexagonal lattice and inserted into a glass tube to form the first stage preform with an outer diameter (OD) of a few $\mathrm{cm}$. The first stage preform is drawn down to a microstructured cane with an OD of a few $\mathrm{mm}$ which is then inserted into another jacketing tube to form the second stage preform. This final preform is then drawn on a fibre drawing tower to produce the final HC-PBGF with an OD ranging from 100-300 microns depending on the operating wavelength ${ }^{7}$.

Longitudinal consistency of both preform and cane over their entire length is required to produce a long length of uniform fibre free from defects/inconsistencies, which can significantly degrade the transmission quality of the fibre ${ }^{3}$. This need for consistency is of paramount importance when considering these fibres as candidates for next generation telecom systems.

\section{Tomographic methods}

CT methods allow observation of the internal structure of preforms, canes and fibres, to investigate their structural uniformity in three dimensions.

Among X-ray, visible light and acoustic tomography methods the first two provide the highest geometric detail ${ }^{8}$ - about $1 \mu \mathrm{m}$ for visible light tomography. Visible light methods are however limited to rather low refractive index changes in the internal structure of the sample, therefore holey or microstructured samples need to be filled with suitable refractive index matching oil, which in most cases is destructive and not reversible. X-ray CT, however, does not require any modification of the sample.

MOF preforms and canes can be analysed by $\mathrm{X}$-ray CT without destroying the sample, unlike conventional methods that requires destructive sample preparation such as polishing. For MOFs, the only conventional method to investigate longitudinal structural changes is cleaving which potentially damages the features of interest in the microstructure. Advances in tomography methods have enabled high resolution imaging of the cross section of fibres as well as along their length. To the best of our knowledge, no other method can offer better resolution in the longitudinal direction.

\section{Results}

Figure 1 shows the result of a CT scan of each key fabrication stage for a typical HC-PBGF. Tomographic imaging of the preform, Fig. 1(a), allows the identification of longitudinal inconsistencies in the arrays of capillaries (i.e. twist, bend or drift). In addition, one should be able to identify contamination (based on shape or material density), and/or failure of any capillaries (cracks). This may enable substantially improved time and cost management of the fibre fabrication process. Fig. 1 (a) shows an example of a preform with some arrangement drift across the preform structure in the very outer ring. In Fig. 1 (b) the $X$-ray CT image shows the excellent consistency and uniformity of the structure within a MOF cane. The voxel size and scan length are $3.35 \mu \mathrm{m}$ and $3.39 \mathrm{~mm}$ respectively. In Fig. 1 (c) an image of a 19-cell HC-PBGF is presented with a quarter cut-away. The voxel size here is set to $0.418 \mu \mathrm{m}$ and the scan length is $407 \mu \mathrm{m}$. In all of these images contamination and structural defects larger than a voxel should be detectable.

Sensitivity to the density of materials is another advantage of this method, which we have explored for the study of contamination within the preforms, canes and fibres. Fig. 2 (a) shows a high-density contaminant in between the outer ring of the microstructure cladding and the jacketing glass in a cane. The feature is consistent with a metal particle, which may have been inadvertently incorporated during the preform assembly.

\section{HC-PBGF Splice}

Fusion splicing is the conventional method of joining two optical fibres together with low loss. The same technology, but with an advanced procedure, can be used to join HC-PBGFs together or to solid fibres. Low loss and robust HC-PBGF-to-HC-PBGF or HC-PBGF to solid fibre splices have been demonstrated ${ }^{9}$ but 
a)

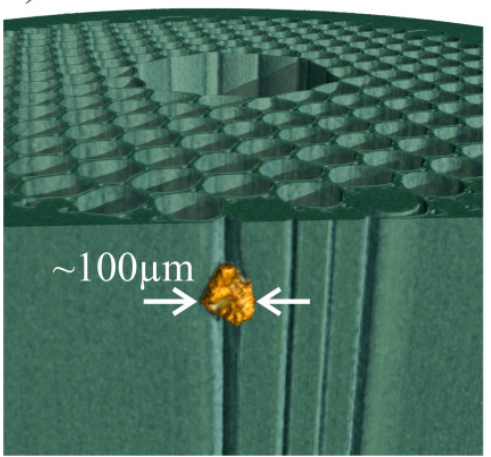

b)

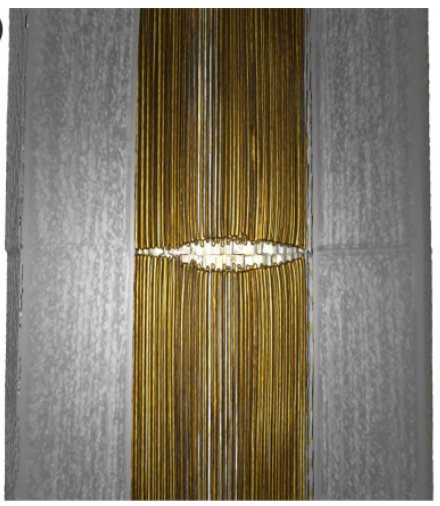

c)

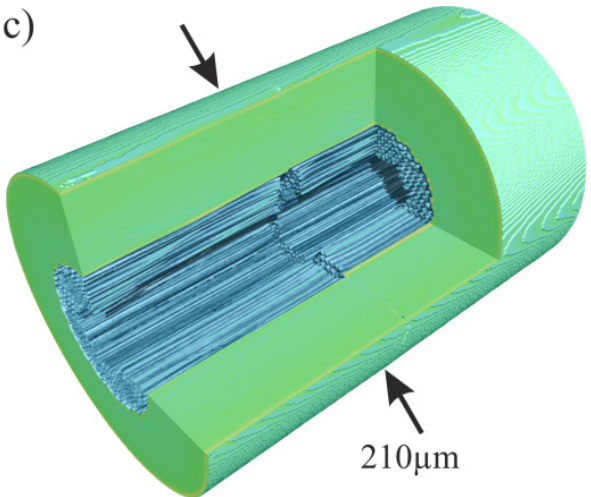

Fig. 2. a) Metal-like contamination in a cane. b) Cavity formation at a splice point. c) Detailed tomographic image of a splice.

careful choice of the splice recipe in order to avoid damage to the microstructure is essential. Some qualitative information on the effect of the splice can be gained e.g. from side imaging of the splice or by carefully breaking and inspecting the splice point, however this is insufficient for the optimisation of the splice process. CT imaging, however, offers the ability to investigate the quality of the bond and to explore the induced structural deformation with unprecedented detail. To illustrate this we used the Xradia Versa with a voxel size of $0.714 \mu \mathrm{m}$ to study the structural changes induced in fusion splicing of two HC-PBGFs. We obtained quantitative data about the impact of the splicing process on the structure of the fibres on either side of a splice. Note that visible light tomography is not possible here because of the high index contrast and impossibility of filling the structure with index matching liquids. A previously reported splice recipe ${ }^{10}$ was used.

Fig. 2 ( $b$ and $c$ ) shows the result of the splice scan where a quarter of the structure is clipped out to expose the internal structure of the fibre. The outer diameter of the fibres is $211 \mu \mathrm{m} \pm$ $0.7 \mu \mathrm{m}$. The position of the joint where the jacketing glass fused together is visible in the centre of the image. The heat during the splice causes the microstructure to retract slightly into the respective fibres leaving a funnel-like cavity in the cladding region of each fibre. The distance between the two microstructure claddings vary between 0 and $21 \mu \mathrm{m}$.

The 3D data set of the splice contains vast amounts of information. In the splice we can observe the impact of cleave quality on the strength of the bond; the uneven features on the cleaved faces caused a gap in the bond between the fibres. Micron scale misalignment of the cladding boundaries is clearly seen in the data. The concentricity of the core-to-core alignment is also measurable. Structural deformations due to the heating can be observed in the data up to $165 \mu \mathrm{m}$ before and after the splice joint. For example, the core expanded from a nominal $38 \mu \mathrm{m}$ to $41 \mu \mathrm{m}$ near the join over this distance scale. Correlation between the structural distortions and the splicing parameters at various stages of the process can help to optimise the splicing recipe.

\section{Conclusions}

We present the first application of X-ray CT to inspect the internal features of HC-PBGFs and their preforms. Moreover, we have applied this non-destructive technique to visualise and measure macroscopic and microscopic deformation within a splice. This technique unveils structural changes induced by the splice process hidden to other inspection methods. Our results illustrate the capability and value of using X-ray CT techniques in the ongoing refinement of various aspects of MOF fabrication and technology.

We wish to thank $\mu$-VIS Centre for Computed Tomography at the University of Southampton. This work was supported by the Royal Society and by the EU FP7-ICT under grant 258033 (MODE-GAP).

\section{References}

[1] S. Jain et al., "Multi-Element Fiber Technology for Space-Division Multiplexing Applications," Opt. Express, Vol. 22, no. 4, p. 3787 (2014)

[2] Z. Lian et al., "Electrical current-driven dual-core optical fiber with embedded metal electrodes," Proc. OFC, Tu3K.3, San Francisco (2014).

[3] S. R. Sandoghchi, et al., "First Investigation of Longitudinal Defects in Hollow Core Photonic Bandgap Fibers," Proc. OFC, M2F.6., San Francisco (2014).

[4] R. Hanke et al., "X-ray based methods for nondestructive testing and material characterization," Nucl. Instrum. Meth. A, Vol. 591, no. 1, p. 14 (2008).

[5] P. R. Shearing et al., "Towards intelligent engineering of SOFC electrodes: a review of advanced microstructural characterisation techniques," Int. Mater. Rev., Vol. 55, no. 6, p. 347, (2010)

[6] F. Poletti et al., "Towards high-capacity fibre-optic communications at the speed of light in vacuum," Nat. Photon., Vol. 7, no. 4, p. 279 (2013).

[7] F. Poletti et al., "Hollow-core photonic bandgap fibers: technology and applications," Nanophotonics, Vol. 2, no. 5-6, p. 315 (2013).

[8] A. D. Yablon, "Multifocus tomographic algorithm for measuring optically thick specimens," Opt. Lett., Vol. 38, no. 21 , p. 4393 (2013).

[9] J. Wooler et al., "Robust Low Loss Splicing of Hollow Core Photonic Bandgap Fiber to Itself," Proc. OFC, OM3I.5, Anaheim (2013).

[10]J. P. Wooler et al., "Overcoming the Challenges of Splicing Dissimilar Diameter Solid-Core and HollowCore Photonic Band Gap Fibers," Proc. WSOF, W3.26, Sigtuna (2013). 